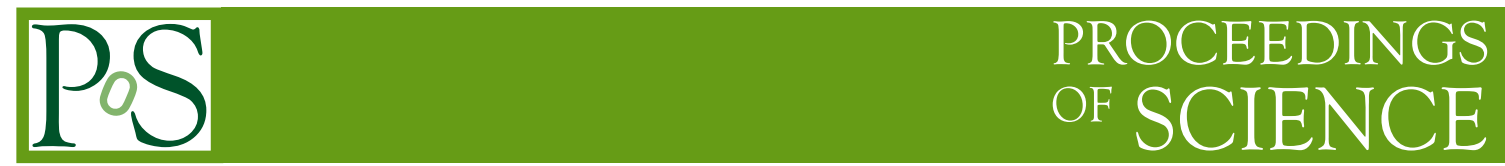

\title{
JLEIC forward detector design and performance
}

\author{
Rik Yoshida*† \\ Jefferson Lab \\ E-mail: ryoshidaejlab.org
}

\begin{abstract}
A major part of the physics program at the Electron-Ion Collider being planned in the US is the exploration of nucleon and nuclear structure. This program means that detection of particles that move in the forward direction and are associated with the beam electrons and protons(ions) is an important part of the detector design as well as of the accelerator and interaction area designs. The current planning of the forward detection for the Jefferson Lab Electron-Ion Collider is discussed.
\end{abstract}

XXIV International Workshop on Deep-Inelastic Scattering and Related Subjects

11-15 April, 2016

DESY Hamburg, Germany

\footnotetext{
* Speaker.

$\dagger^{\dagger}$ on behalf of the JLEIC Detector and IR Study Group
} 


\section{Jefferson Lab Electron-Ion Collider}

The Jefferson Lab proposal for the Electron-Ion Collider (JLEIC) builds on the existing CEBAF accelerator as a full-energy injector and adds a new ion complex to achieve collisions of electrons of energies up to $10 \mathrm{GeV}$ with protons of energies up to $100 \mathrm{GeV}$, as well as ions of energies of $100 \times(Z / A) \mathrm{GeV} /$ nucleon at luminosities of $10^{34} \mathrm{~cm}^{-2} \mathrm{~s}^{-1}$ (fig. 1). The electron and ion rings are stacked in an innovative figure- 8 design that allows protons and light-ion polarization in excess of $70 \%$. The two interaction points (IP) sit in the straight sections relatively closer to the ion bend arc and far from the electron bend arc in order to avoid the ion related beam-gas background and synchrotron radiation respectively [1].

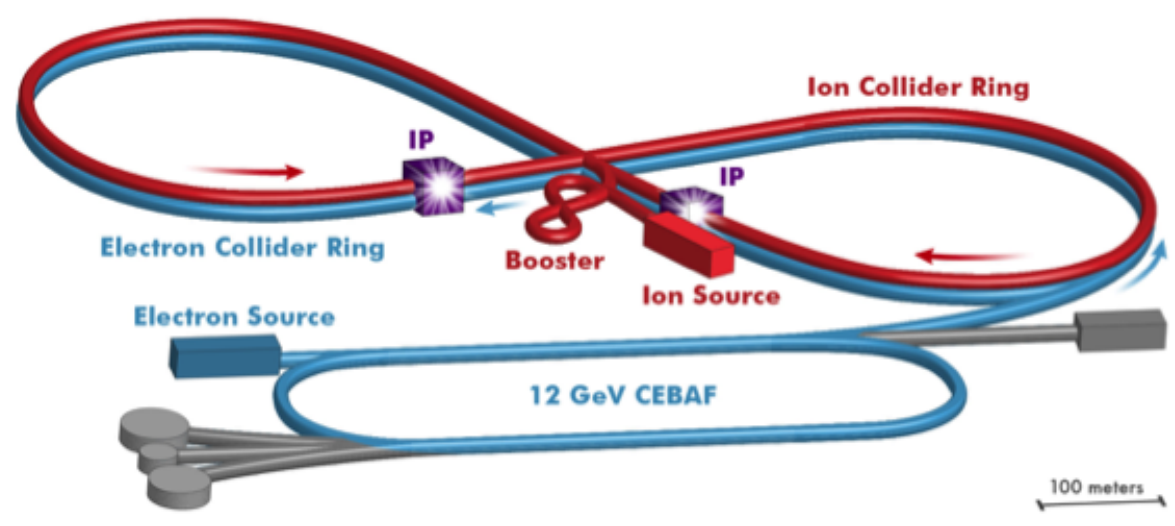

Figure 1: The envisioned JLEIC facility: the continuous electron accelerator facility (CEBAF) at $12 \mathrm{GeV}$ electron beam is in operation as of this writing. The plans add an ion facility and an electron collider ring to the existing infrastructure.

\section{Total Acceptance Detector}

The basic physics process at the Electron-Ion Collider (EIC) is Deep Inelastic Scattering (DIS) in which the electron collides with a parton within an ion in a hard collision. We can, qualitatively, define three classes of particles in the final state. 1. The scattered electron 2. Particles associated with the initial state ion. 3. Particles associated with the struck parton.

All three types of final state particles carry information about the ion. Therefore it is essential that the Interaction Region (IR) and the detector at the EIC be designed so that all three types of particles are measured at as close to $100 \%$ acceptance and with the necessary resolutions. We define the concept of a total acceptance detector as one that achieves close to $100 \%$ acceptance for all three types of particles.

The difficulty in achieving good acceptance in the forward regions at a collider has to do with the accelerator elements needed to deliver the colliding beams. To first order, the luminosity at the IP is inversely proportional to the distance between the final focus ion quadrupoles. Thus, the statistical uncertainty of the measurements at the Central Detector scales as $\sqrt{(\text { distance })}$. On the 
other hand, the beam elements obstruct the acceptance at shallow angles with respect to the beam axis. The closer to the IP they are, the more problematic they are for forward particle acceptance. Also, the solenoidal field used in the central detector region to measure the high Pt particles in the central detector is not effective in determining the momenta of particles moving parallel to the beam direction, and additional fields are needed. The JLEIC IR and detector concept, shown schematically in fig. 2 is designed to overcome the measurement difficulties posed by the beam elements. The ion beam and electron beams cross at a relatively large angle of 50mr at the IP. High luminosity is preserved through the use of crab cavities. This angle moves the ion beam away from the electron beam elements and makes room for dipoles located just downstream of the central detector area. The dipoles serve two purposes. First they further move the beams apart so that there is $1 \mathrm{~m}$ distance between the two beams $30 \mathrm{~m}$ away from the IP, making room for detectors. Second, the dipoles (dipole systems) allow momentum analyses of the particles with small transverse momentum with respect to the beams. The particles with large transverse momenta are analyzed using the solenoidal field in the central detector.

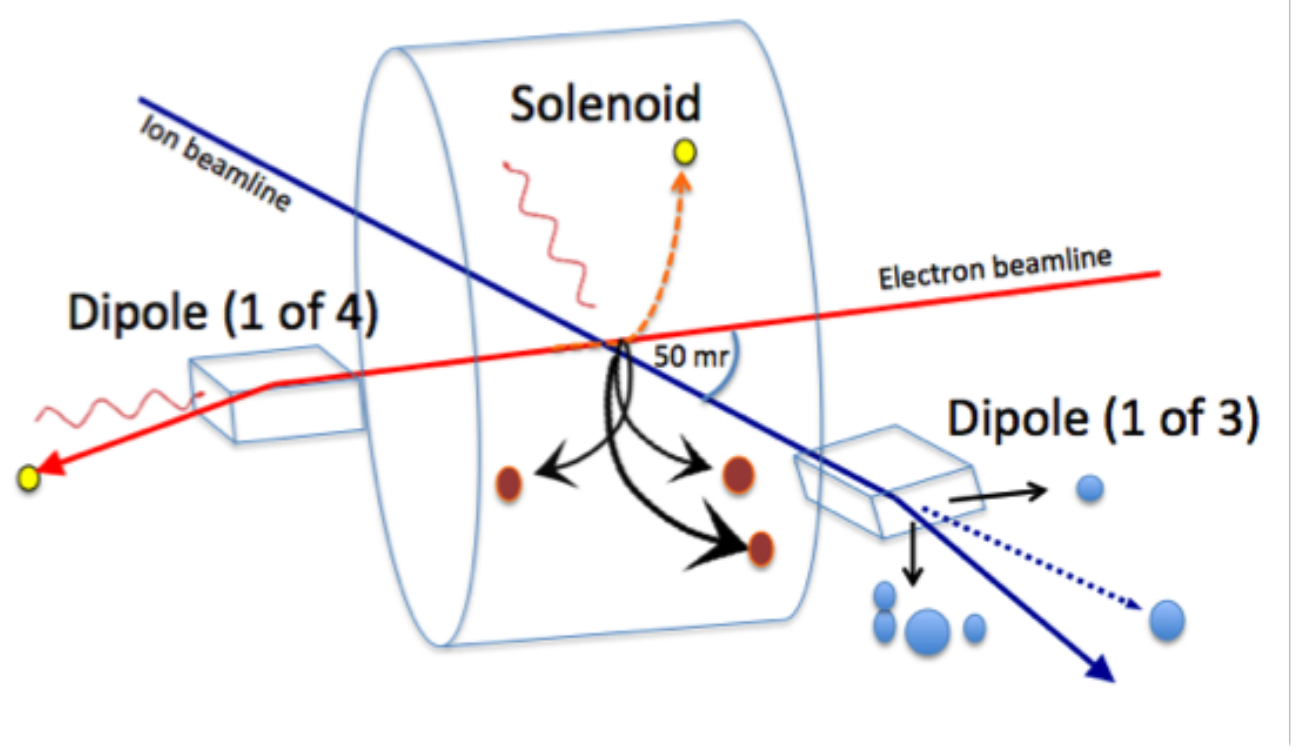

Figure 2: A total acceptance detector at the EIC: All final state particles, including those associated with the beam proton(ion) are accepted and measured at high precision.

\section{Forward Ion Detector}

Crucial information on hadron structure is carried by particles that do not emerge from the beam envelope within the coverage of the central detector. Broadly speaking, there are two types of forward final state particles need to be reconstructed. The first type of forward particles comes from interactions in which the beam particle receives a large transverse momentum kick and fragment into many parts. These particles typically retain a velocity similar in magnitude, but significantly different from that of the beam particle, and may have very different charge-to-mass ratios. Such particles will separate relatively rapidly from the beam. An example of such a particle is a 
forward proton from a deuteron-electron DIS shown in fig 3Right. Measurements of such particles are important in understanding the nucleon-nucleon interactions in nuclei, as well as, in case of deuterons, determining the structure of neutrons. The second type is the (hadron) beam particle that stays intact during the collision and only loses a small fraction of their momentum and acquires a small transverse momentum $\left(p_{T}\right)$. These particles are leading protons or ions in non-dissociative diffractive interactions (see fig. 4Left), and will have a trajectory that is close to the proton (or the ion) beam.
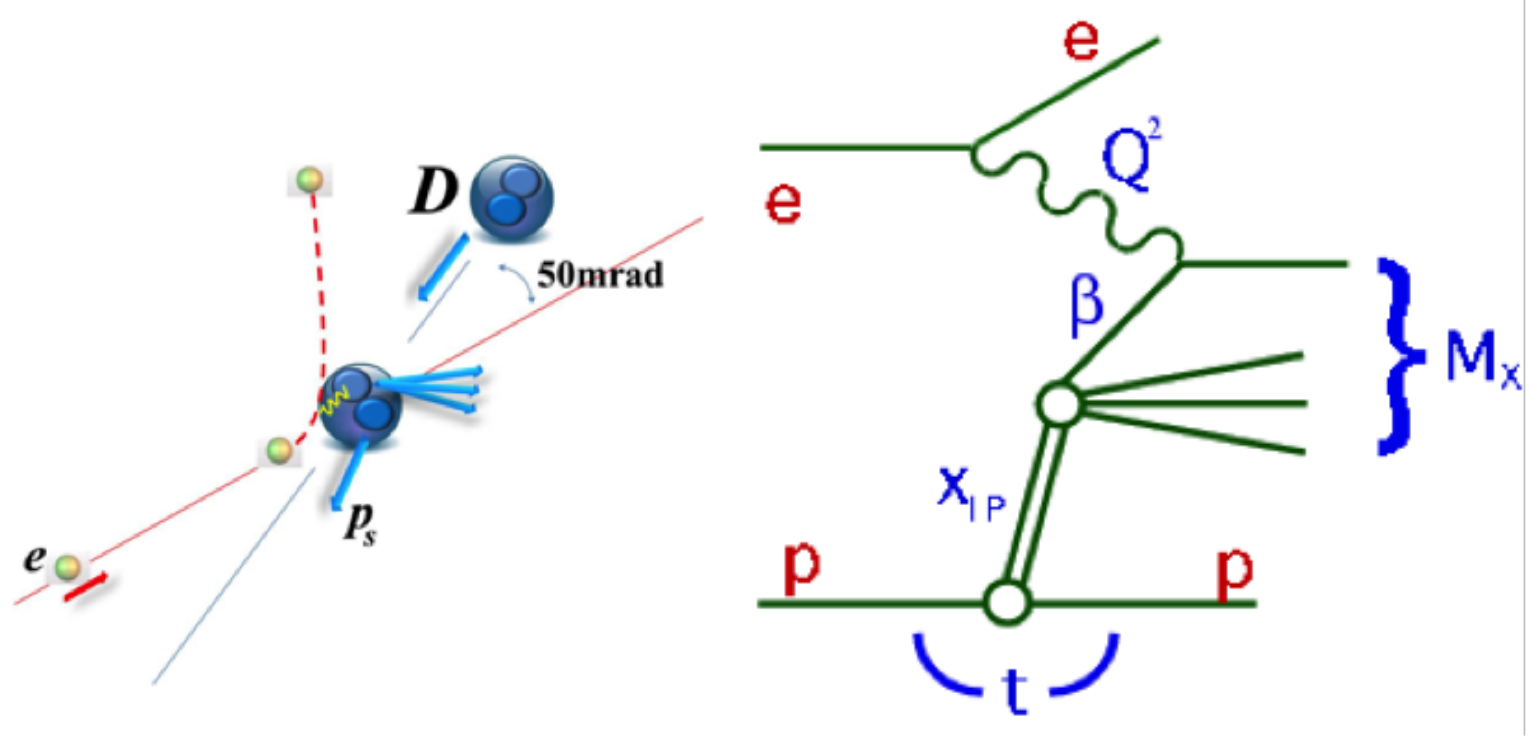

Figure 3: Left) Electron-deuteron collision at the EIC: the collision probes the neutron structure. Final state proton with momentum $p_{s}$ needs to be measured. Right) Diffractive deep inelastic scattering: the 4-momentum transfer $t$ at the proton vertex is small. The outgoing proton needs to be measured.

There are two detector regions downstream of the ion beam after the central detector. The first area is between the first dipole downstream of the interaction point in the ion beam direction and the final focus ion quadrupole magnets (FFQs), see fig. 4Right; this region will be called the forward detection region and is about $50 \mathrm{~cm}$ longitudinally. The first dipole provides about 1 $T-m$ of field and thus gives moderate analyzing power for those particles detected in the forward region. After the FFQs, second and third long dipoles (20 $T$ - $m$ combined) provides the possibility of high-resolution measurements for those particles that pass through the apertures of the FFQs. The area, about $30 \mathrm{~m}$ longitudinally, surrounding the two dipoles is the, second, or high-resolution, forward ion detection region. These two detector regions together with the detector systems around the beampipes in the central detector provides essentially $100 \%$ coverage for final state particles associated with the ion (proton) beam particle. The leading protons and ions in non-dissociative diffractive interactions, whose measurements are an important part of the EIC physics program, will be mainly measured in the high-resolution forward detector region. In order to maximize the acceptance and resolution for these particles, the optics of the beams are arranged such that there is a secondary focus of the beam at $42 \mathrm{~m}$ downstream of the interaction point. At the same point, the dispersion is designed to have a maximum. A detector system at this point will have maximum acceptance for final state particles whose momenta are close to that of the beam particles. 
Figure 4Right shows the acceptance for protons generated at the interaction point having energies of $100 \times x_{L} \mathrm{GeV}$ and having $p_{T}$ up to $1 \mathrm{GeV}$, where $x_{L}$ is the fraction of the initial longitudinal momentum carried by the final state proton. (i.e. momentum range expected for leading protons for non-dissociative interaction at a proton beam energy of $100 \mathrm{GeV}$ ). The acceptance is $100 \%$ in all of this area except for a diagonal region shadowed by the aperture of the FFQs. Even in this area, the acceptance is mostly above 50\%. The lower right triangular area corresponds to the kinematics that will be measured in the high-resolution forward detection region. The large aperture of the FFQs also allows a large acceptance for zero-degree calorimeter placed at around $30 \mathrm{~m}$ downstream for neutron detection.
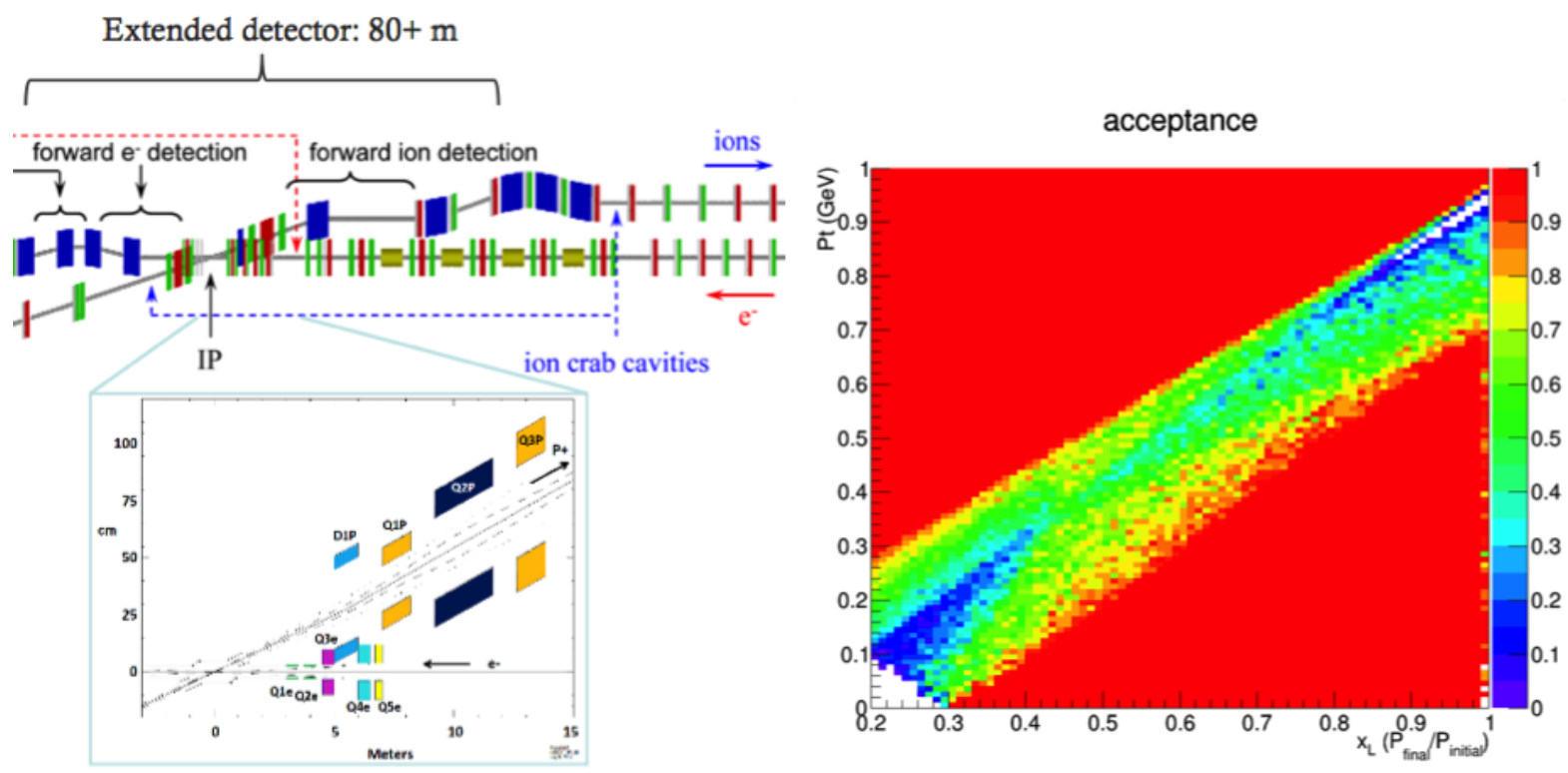

Figure 4: Left) Accelerator elements in the IR and in the downstream ion area where forward ion detectors will be placed. The dark blue elements are dipoles where as red and green elements are beam quadrupoles. The insert shows the details near the IR. The element labeled D1p is the first dipole, which are followed by the three FFQs. Right) The acceptance for the leading proton in diffractive DIS in the forward and high-resolution forward detector areas as a function of $x_{L}$ and $p_{t}$.

\section{Conclusion}

The Jefferson Lab Electron Ion Collider (JLEIC) interaction region and detectors have been designed to accommodate a total acceptance detector in which all final particles, including those associated with the beam particles are detected with close to $100 \%$ acceptance and measured with good resolution. Some of the design philosophy and characteristics of this design has been discussed.

\section{References}

[1] S. Abeyratne, et al., "MEIC Design Summary", arXiv:1504.07961 (2016) 